\title{
Formulation and In-Vitro Evaluation of Fast Dissolving Tablets of Anti-Ulcer Drugs
}

\author{
K S Shrilatha ${ }^{1}$, Senthilkumar K*2, Likhith H M², Birendra Prasad Gupta ${ }^{\mathbf{1}}$ \\ 1.Department of Pharmaceutics, $R R$ college of pharmacy, Banglore-560090 \\ 2.Sri Adichunchanagiri College Of Pharmacy, Adichunchanagiri University, B G Nagar- \\ 571448, Karnataka
}

\section{ABSTRACT}

Pantoprazole is a proton pump inhibitor that decreases the amount of acid produced in the stomach mainly used to treat erosive esophagitis and other condition involving excess stomach acid such as Zollinger-Ellison syndrome. The objective of present study was to Formulate and Evaluate fast dissolving tablets of Pantoprazole sodium. In the preformulation study, IR Spectra of pure drug and with different polymers showed no interaction (no shift in peak). To enhance faster disintegration rate, super disintigrants such as croscarmallose sodium, Crospovidone and Sodium starch glycolate were tried. To evaluate their role in fast dispersion, they were used in different concentrations hence in the present study 9 formulations were prepared. The prepared tablets were subjected to various parameters like uniformity of weight, hardness, friability, drug content, water absorption ratio, wetting time, in vitro disintegration time and in vitro dissolution studies. The effect of different super disintigrants over the Drug release profile was investigated. In the study, all powder blends showed good flow ability (angle of repose below $30^{\circ}$ ), bulk density in the range between $0.33-0.37 \mathrm{~g} / \mathrm{cm}^{3}$ tapped density in the range between 0.34 and 0.39 $\mathrm{g} / \mathrm{cm}^{3}$, and the compressibility index was found to be between 5.83 and $9.98 \%$, which ensures the blend that may be suitable for direct compression in to tablets. In vitro disintegration time for all formulation batches i.e. F-1 to F-9 showed wide variation in the range between 8.78 .64 to 19.35 seconds and \% Drug dissolved at 30 seconds. The prepared tablets exhibited satisfactory physico-chemical characteristics. The prepared formulations containing superdisintegrants, Crospovidone Along with microcrystalline cellulose showed faster dispersion and dissolution profile as compared with other two superdisintegrants containing formulations. Moreover, the study revealed Crospovidone showed satisfactory results than the superdisintegrants like croscarmilose sodium and sodium starch glycolate.

Keywords: Pantoprazole, Fast dissolving tablets, polymers, in-vitro drug release. 


\section{INTRODUCTION}

Fast dissolving tablet is a solid dosage form that dissolves or disintegrates within a minute in the oral cavity without the need of water and has a pleasant taste. FDT is also known as orally disintegrating tablet, fast-dissolving tablet, fast-melting tablet or mouth melting tablet. Orally disintegrating tablets are also called as Orodispersible tablet, quick disintegrating tablets Fast Disintegrating tablets. Fast disintegrating tablets, fast dissolving tablets, rapid dissolving tablets, porous tablets and rapid melt. European pharmacopoeia has used the term orodispersible tablet for tablet that disperse readily and within $3 \mathrm{~min}$ in mouth before swallowing.

When water is not available, in the case of motion sickness (kinetosis) and sudden episodes of coughing during the common cold, allergic conditions and bronchitis for these reasons, tablets which can rapidly dissolve or disintegrate in the oral cavity have attracted a great deal of attention. Rapidly dissolving or disintegrating tablets are not only indicated for people who have swallowing difficulties, but also are ideal for active people.

Pantoprazole is a proton pump inhibitor that decreases the amount of acid produced in the stomach mainly used to treat erosive esophagitis and other condition involving excess stomach acid such as Zollinger-Ellison syndrome.

\section{MATERIALS AND METHOD}

\section{Preformulations Studies}

Standard calibration curve of pantoprazole sodium: Pantoprazole exhibits peak absorbance at $288 \mathrm{~nm}$ in Phosphate buffer having pH 6.4 represents the maximum absorbance $(\lambda \mathrm{max})$ of the drug.

\section{Procedure:}

$\lambda \max$ of pantoprazole $=288 \mathrm{~nm}$

Beer's range of pantoprazole $=1-5 \mu \mathrm{g} / \mathrm{m}$

\section{Preparation of standard solution:}

$1^{\circ}$ stock: $100 \mathrm{mg}$ of pantoprazole was weighed accurately into $100 \mathrm{ml}$ volumetric flask and the volume was made up with Phosphate buffer.

$2^{\circ}$ stock: Pipette $1 \mathrm{ml}$ of the solution into another $100 \mathrm{ml}$ volumetric flask and the volume was made with the water. (i.e.: $10 \mu \mathrm{g} / \mathrm{ml}$ in water.)

Preparation of working standard solution: Aliquots of standard solution $1 \mathrm{ml}, 2 \mathrm{ml}, 3 \mathrm{ml}, 4 \mathrm{ml}, 5 \mathrm{ml}$ were pipette into $10 \mathrm{ml}$ volumetric flasks. The volume made up with water. The absorbance of each concentration was measured at $288 \mathrm{~nm}$ using Phosphate buffer as a blank. 
Table 1: Standard calibration data of Pantoprazole in phosphate buffer (pH 6.4)

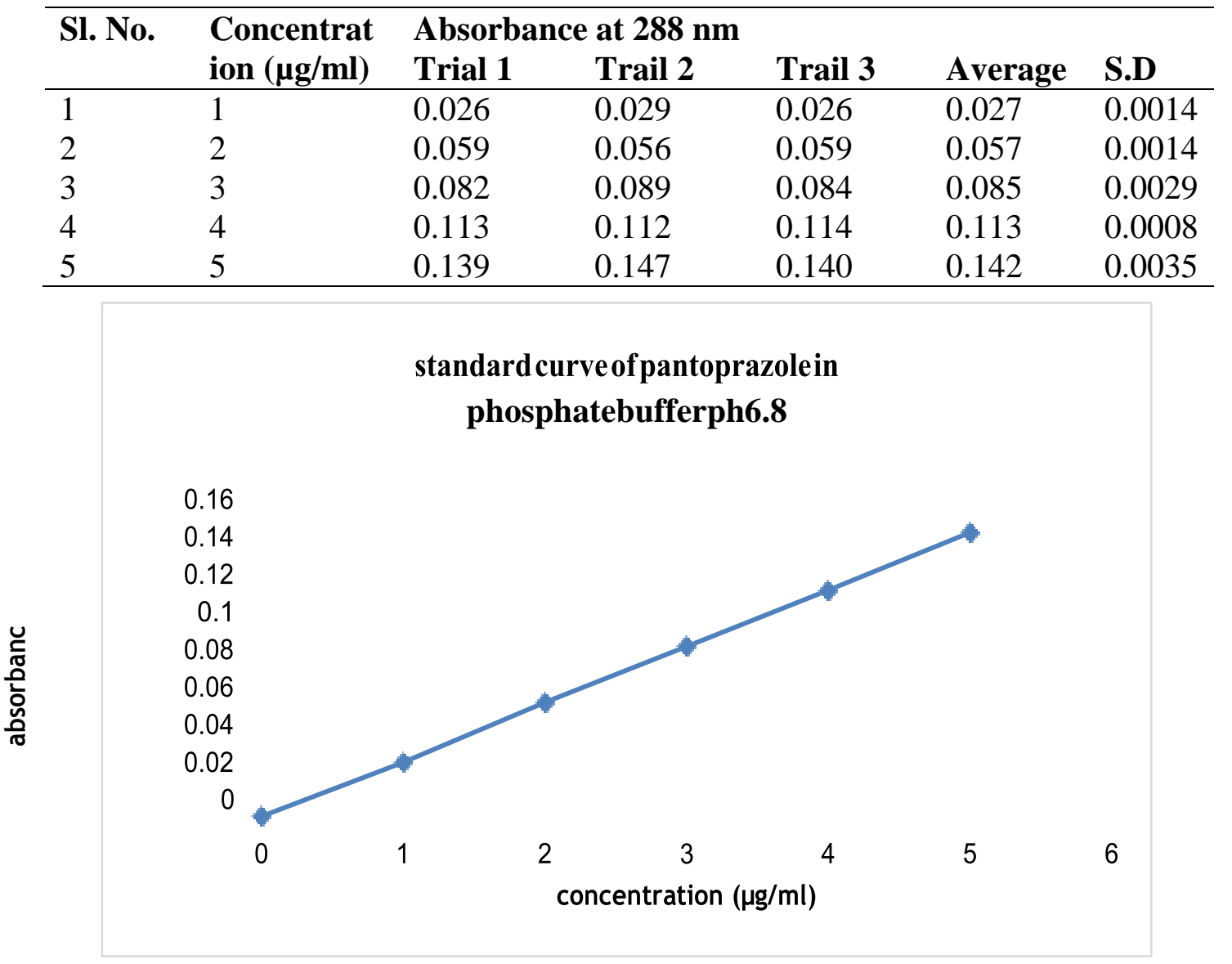

Figure:1 Standard Curve of Pantoprazole in Phosphate buffer $\left(\mathbf{P}^{\mathrm{H}} 6.8\right)$

\section{Formulation}

The drug was mixed with proper portion of superdisintegrants. Care was taken to confirm the proper mixing of drug and superdisintegrants. Then the mixture is passed through sieve No.44, after addition of the other excipients. The mixture was blended with lubricating agent (magnesium stearate) and glidant. Finally the blend was subjected for compression using 10mm on Rimek mini press 10 station machines.

Table 2 Formulations Pantoprazole Sodium

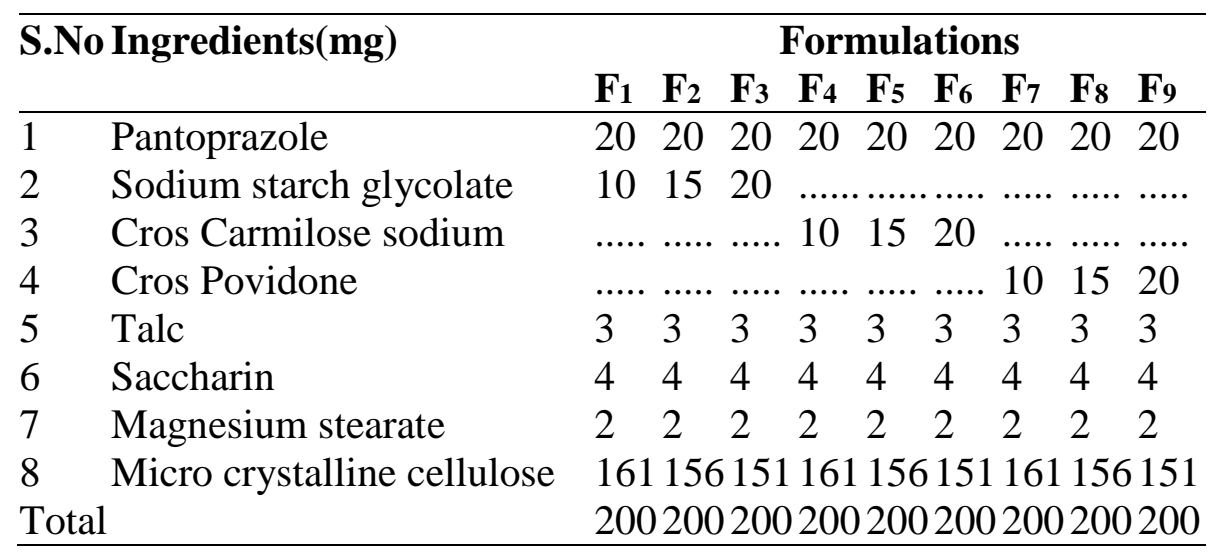




\section{RESULTS AND DISCUSSION}

Table 3: Pre-compression parameters of pantoprazole powder mixture

\begin{tabular}{|c|c|c|c|c|c|}
\hline \multicolumn{6}{|l|}{ Fori } \\
\hline code & $\operatorname{repose}(\theta)$ & $\left(\mathrm{gm} / \mathrm{cm}^{3}\right)$ & $\left(\mathrm{gm} / \mathrm{cm}^{3}\right)$ & bility & Ratio \\
\hline$\overline{\mathrm{F} 1}$ & $29.02 \pm 1.32$ & $20.35 \pm 0.012$ & $0.39 \pm 0.005$ & $9.98 \pm 0.78$ & $1.11 \pm 0.009$ \\
\hline $\mathrm{F} 2$ & $28.15 \pm 1.97$ & $70.33 \pm 0.005$ & $0.36 \pm 0.008$ & $7.98 \pm 0.16$ & $1.08 \pm$ \\
\hline F3 & $27.06 \pm 1.19$ & $90.36 \pm 0.017$ & $0.38 \pm 0.007$ & $5.99 \pm 0.04$ & $1.06 \pm 0.012$ \\
\hline $\mathrm{F} 4$ & $27.6 \pm 1.80$ & $0.35 \pm 0.003$ & $0.37 \pm 0.003$ & $6.06 \pm 0.07$ & $1.06 \pm 0.007$ \\
\hline F5 & $29.02 \pm 1.48$ & $80.36 \pm 0.012$ & $0.39 \pm 0.002$ & $8.10 \pm 0.16$ & $1.08 \pm 0.003$ \\
\hline F6 & $25.88 \pm 1.17$ & $70.35 \pm 0.015$ & $0.36 \pm 0.001$ & $14.04 \pm 0.23$ & $1.16 \pm 0.017$ \\
\hline F7 & $27.70 \pm 1.75$ & $50.33 \pm 0.020$ & $0.34 \pm 0.002$ & $6.89 \pm 0.09$ & $1.07 \pm 0.015$ \\
\hline F8 & $26.36 \pm 1.48$ & $80.37 \pm 0.007$ & $0.39 \pm 0.003$ & $5.83 \pm 0.03$ & $1.06 \pm 0.005$ \\
\hline F9 & $27.70 \pm 1.38$ & $80.33 \pm 0.014$ & $0.35 \pm 0.002$ & $5.86 \pm 0.02$ & $1.06 \pm 0.006$ \\
\hline
\end{tabular}

Table 4: Evaluation of post compression parameters of fast dissolving tablet containing Pantoprazole:

\begin{tabular}{lllll}
\hline \multicolumn{4}{l}{ Formulation Thickness(mm)Wetting timeWater abs.In vitro dispersion } \\
& $3.02 \pm 0.03$ & $32 \pm 2.13$ & $86 \pm 3.12$ & $26 \pm 0.8$ \\
$\mathrm{~F}_{1}$ & $3.05 \pm 0.02$ & $27 \pm 3.7$ & $84 \pm 2.98$ & $23 \pm 0.2$ \\
$\mathrm{~F}_{2}$ & $3.12 \pm 0.02$ & $28 \pm 4.1$ & $87 \pm 3.19$ & $21 \pm 0.8$ \\
$\mathrm{~F}_{3}$ & $3.17 \pm 0.12$ & $22 \pm 3.41$ & $91 \pm 0.02$ & $18 \pm 1.2$ \\
$\mathrm{~F}_{4}$ & $3.08 \pm 0.8$ & $21 \pm 3.1$ & $93 \pm 1.94$ & $17 \pm 0.8$ \\
$\mathrm{~F}_{5}$ & $3.1 \pm 0.17$ & $19 \pm 1.2$ & $95 \pm 2.12$ & $19 \pm 0.59$ \\
$\mathrm{~F}_{6}$ & $3.1 \pm 1.2$ & $29 \pm 2.87$ & $76 \pm 2.10$ & $28 \pm 1.2$ \\
$\mathrm{~F}_{7}$ & $3.05 \pm 0.8$ & $30 \pm 3.78$ & $74 \pm 1.20$ & $26 \pm 0.22$ \\
$\mathrm{~F}_{8}$ & $3.2 \pm 0.12$ & $28 \pm 1.28$ & $78 \pm 2.28$ & $25 \pm 0.58$ \\
$\mathrm{~F}_{9}$ & &
\end{tabular}

\section{FTIR STUDIES}

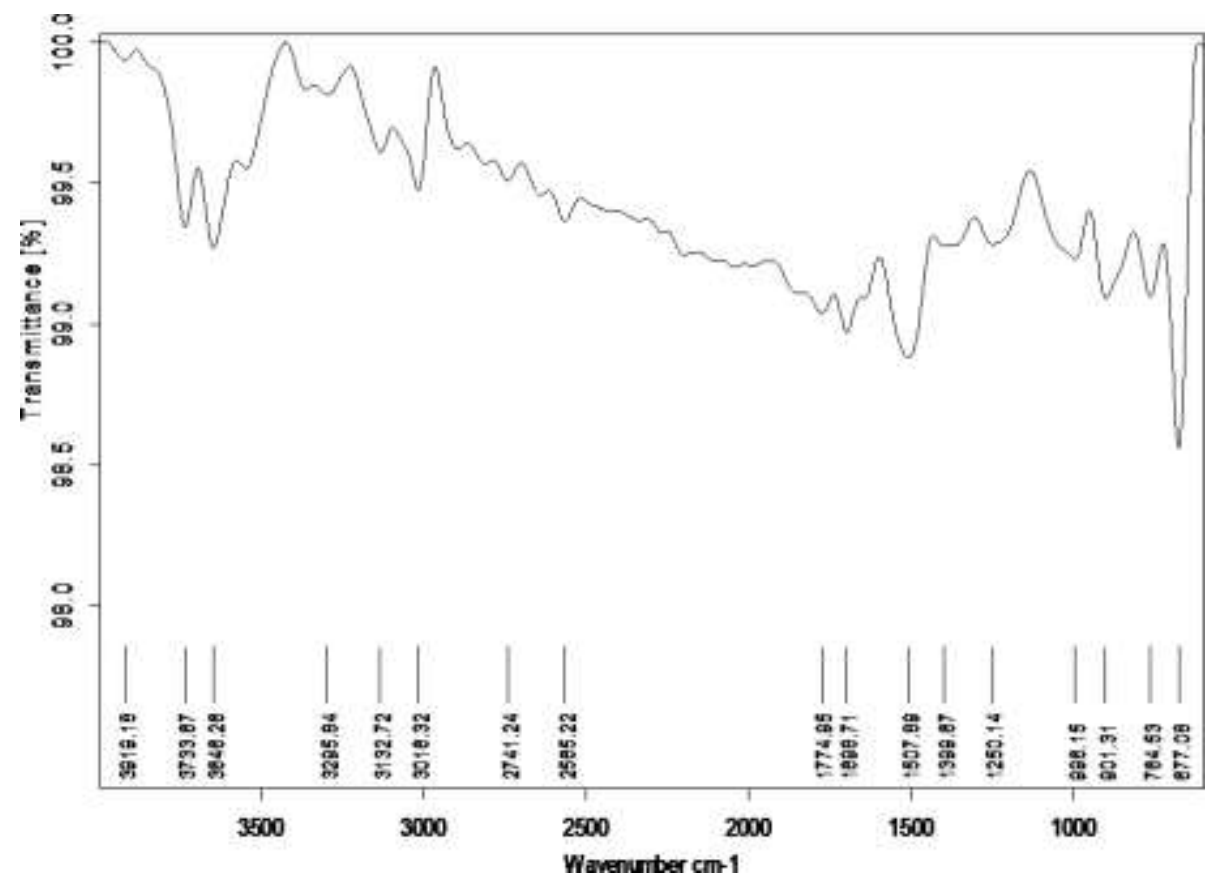

Figure 2: FTIR of Pure Pantoprazole 


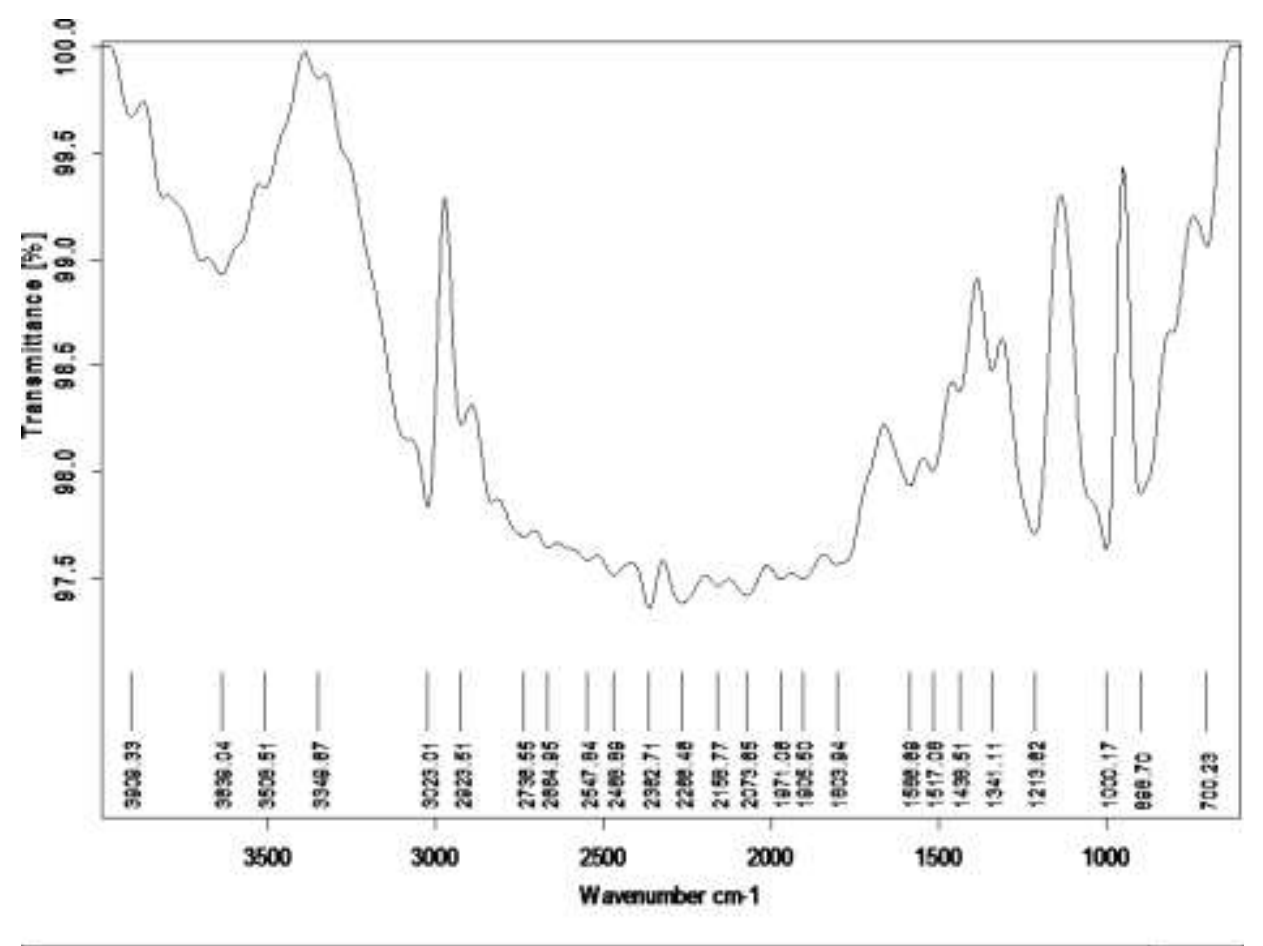

Figure 3: FTIR of Pantoprazole. + Cross Povidone.

Table 5: In vitro dissolution study of formulations (F1)

Weight of the tablet $=204 \mathrm{mg}$. Amount of drug present $=19.4 \mathrm{mg}$

\begin{tabular}{lllllllll}
\hline $\begin{array}{l}\text { Time } \\
(\mathbf{m i n})\end{array}$ & Abs & $\begin{array}{l}\text { Concentration } \\
(\boldsymbol{\mu g} / \mathbf{m l})\end{array}$ & $\begin{array}{l}\text { D.F. } \\
\text { (n) }\end{array}$ & $\begin{array}{l}\text { Amount } \\
\text { in 5ml }\end{array}$ & $\begin{array}{l}\text { Amount } \\
\text { in 900ml }\end{array}$ & CLA & CDR & \% CDR \\
\hline 0 & 0 & 0 & 0 & 0 & 0 & 0 & 0 & 0 \\
2 & 0.0269 & 0.9438 & 10 & 0.04719 & 8.494 & 0 & 8.494 & 43.78 \\
4 & 0.0356 & 1.2491 & 10 & 0.06245 & 11.2491 & 0.04719 & 11.2890 & 58.14 \\
6 & 0.0489 & 1.7157 & 10 & 0.08578 & 15.4413 & 0.10964 & 15.5509 & 80.15 \\
8 & 0.0542 & 1.9017 & 10 & 0.09508 & 17.1153 & 0.19542 & 17.3107 & 89.23 \\
10 & 0.0571 & 2.0035 & 10 & 0.1001 & 18.0315 & 0.2905 & 18.322 & 94.44 \\
\hline
\end{tabular}

Table 6: In vitro dissolution study formulations F2

Weight of the tablet $=205 \mathrm{mg}$. Amount of drug present $=19.6 \mathrm{mg}$

\begin{tabular}{lllllllll}
\hline $\begin{array}{l}\text { Time } \\
(\mathbf{m i n})\end{array}$ & Abs & $\begin{array}{l}\text { Concentration } \\
(\boldsymbol{\mu g} / \mathbf{m l})\end{array}$ & $\begin{array}{l}\text { D.F. } \\
\text { (Amount }\end{array}$ & $\begin{array}{l}\text { Amount } \\
\text { in 5ml }\end{array}$ & $\begin{array}{l}\text { CLA } \\
\text { in 900ml }\end{array}$ & CDR & \% CDR \\
\hline 0 & 0 & 0 & 0 & 0 & 0 & 0 & 0 & 0 \\
2 & 0.0281 & 0.9859 & 10 & 0.04929 & 8.8731 & 0 & 8.8731 & 45.27 \\
4 & 0.0371 & 1.3017 & 10 & 0.06508 & 11.7153 & 0.04929 & 11.7645 & 60.02 \\
6 & 0.0498 & 1.7473 & 10 & 0.08736 & 15.7257 & 0.1143 & 15.840 & 80.81 \\
8 & 0.0531 & 1.8631 & 10 & 0.0931 & 16.7679 & 0.9879 & 17.7558 & 90.59 \\
10 & 0.0584 & 2.0491 & 10 & 1.0245 & 18.4419 & 1.081 & 19.5229 & 99.60 \\
\hline
\end{tabular}


Gupta et al.,

Table 7: In vitro dissolution study of formulations F3

Weight of the tablet $=206 \mathrm{mg}$. Amount of drug present $=19.72 \mathrm{mg}$

\begin{tabular}{lllllllll}
\hline $\begin{array}{l}\text { Time } \\
(\mathbf{m i n})\end{array}$ & Abs & $\begin{array}{l}\text { Concentration } \\
(\boldsymbol{\mu g} / \mathbf{m l})\end{array}$ & $\begin{array}{l}\text { D.F. } \\
\text { (n) 5ml }\end{array}$ & $\begin{array}{l}\text { Amount } \\
\text { in }\end{array}$ & $\begin{array}{l}\text { Amount } \\
\text { in 900ml }\end{array}$ & CLA & CDR & \%CDR \\
\hline 0 & 0 & 0 & 0 & 0 & 0 & 0 & 0 & 0 \\
2 & 0.0312 & 1.0947 & 10 & 0.0547 & 9.8523 & 0 & 9.8523 & 49.96 \\
4 & 0.0361 & 1.2666 & 10 & 0.0633 & 11.3994 & 0.0547 & 11.4541 & 58.08 \\
6 & 0.0501 & 1.7578 & 10 & 0.0878 & 15.8202 & 0.118 & 15.9382 & 80.82 \\
8 & 0.0539 & 1.8912 & 10 & 0.0945 & 17.0208 & 0.2058 & 17.2266 & 87.35 \\
10 & 0.0594 & 2.0842 & 10 & 0.1042 & 18.7578 & 0.3003 & 19.0581 & 96.64 \\
\hline
\end{tabular}

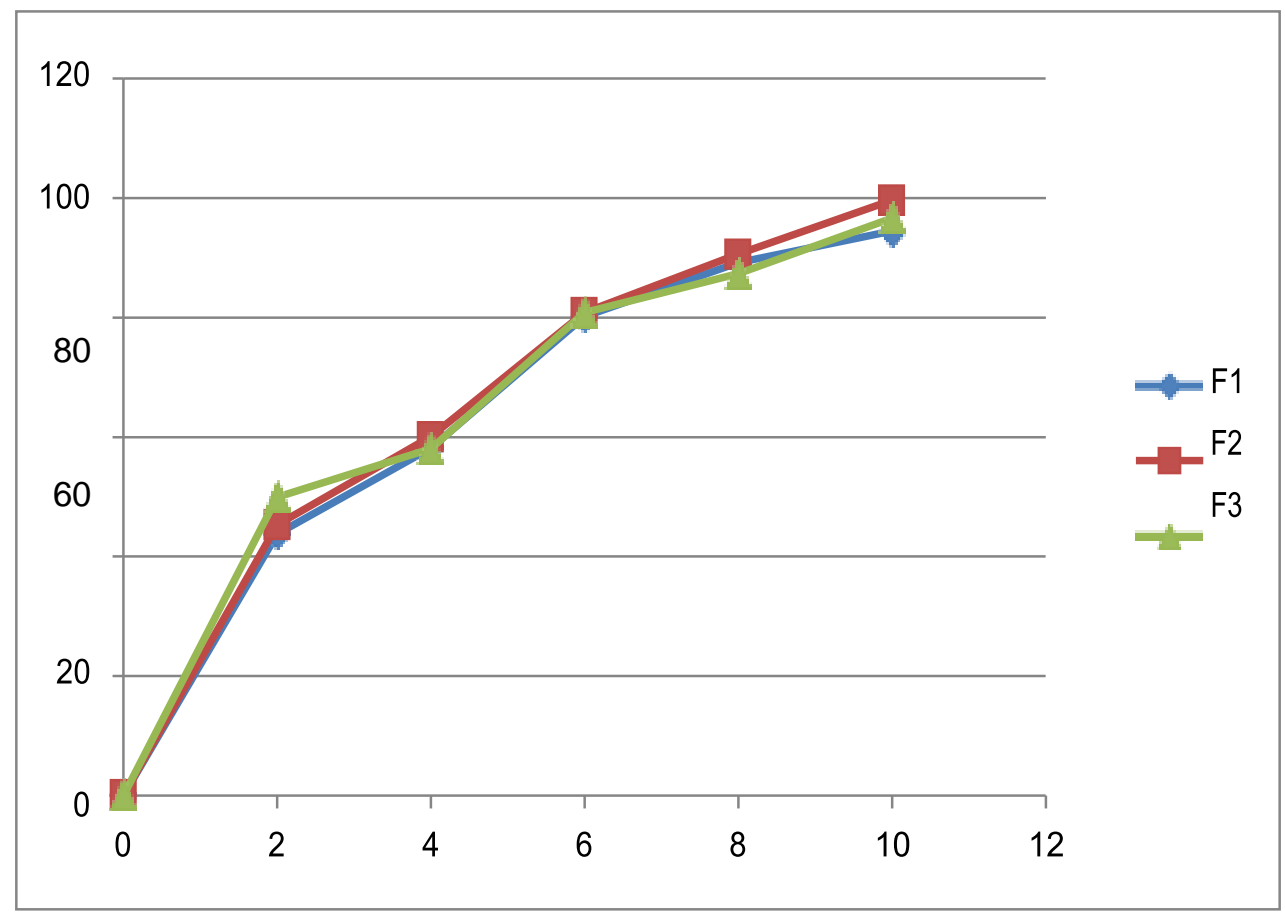

Figure 10: In vitro drug release for $F 1, F 2$ and $F 3$

Table 8: In vitro dissolution study of formulations F4

Weight of the tablet $=202 \mathrm{mg}$. Amount of drug present $=19.56 \mathrm{mg}$

\begin{tabular}{lllllllll}
\hline $\begin{array}{l}\text { Time } \\
(\mathbf{m i n})\end{array}$ & Abs & $\begin{array}{l}\text { Concentration } \\
(\boldsymbol{\mu g} / \mathbf{m l})\end{array}$ & D.F. & $\begin{array}{l}\text { Amount in } \\
\mathbf{5 m l}\end{array}$ & $\begin{array}{l}\text { Amount } \\
\mathbf{9 0 0 m l}\end{array}$ & CLA & CDR & \% CDR \\
\hline 0 & 0 & 0 & 0 & 0 & 0 & 0 & 0 & 0 \\
2 & 0.0271 & 0.9508 & 10 & 0.0475 & 8.5572 & 0 & 8.5572 & 43.74 \\
4 & 0.0301 & 1.0561 & 10 & 0.0528 & 9.50 & 0.475 & 9.9799 & 51.02 \\
6 & 0.0467 & 1.6385 & 10 & 0.0819 & 14.7465 & 0.5278 & 15.2743 & 78.08 \\
8 & 0.0514 & 1.8035 & 10 & 0.0901 & 16.2315 & 0.6097 & 16.8412 & 86.10 \\
10 & 0.0571 & 2.0035 & 10 & 0.1001 & 18.0315 & 1.5107 & 19.5422 & 99.9 \\
\hline
\end{tabular}


Gupta et al.,

Am. J. Pharm Health Res 2020;8(2)

ISSN: 2321-3647

Table 9: In vitro dissolution study of formulation F5

Weight of the tablet $=203 \mathrm{mg}$. Amount of drug present $=19.8$

\begin{tabular}{lllllllll}
\hline $\begin{array}{l}\text { Time } \\
(\mathbf{m i n})\end{array}$ & Abs & $\begin{array}{l}\text { Concentration } \\
(\boldsymbol{\mu g} / \mathbf{m l})\end{array}$ & D.F. & $\begin{array}{l}\text { Amount in } \\
\mathbf{5 m l}\end{array}$ & $\begin{array}{l}\text { Amount in } \\
\mathbf{9 0 0 m l}\end{array}$ & CLA & CDR & \% CDR \\
\hline 0 & 0 & 0 & 0 & 0 & 0 & 0 & 0 & 0 \\
2 & 0.0279 & 0.9789 & 10 & 0.0489 & 8.8101 & 0 & 8.8101 & 44.49 \\
4 & 0.0312 & 1.0947 & 10 & 0.0547 & 9.8523 & 0.0489 & 9.9012 & 50 \\
6 & 0.0498 & 1.7473 & 10 & 0.0873 & 15.72 & 0.1036 & 15.8236 & 79.91 \\
8 & 0.0542 & 1.9017 & 10 & 0.0950 & 17.1153 & 0.1909 & 17.3062 & 87.40 \\
10 & 0.0571 & 2.0035 & 10 & 0.1001 & 18.0315 & 0.2859 & 18.3174 & 92.51 \\
\hline
\end{tabular}

Table 10: In vitro dissolution study of formulations F6

Weight of the tablet $=203 \mathrm{mg}$. Amount of drug present $=19.76 \mathrm{mg}$

\begin{tabular}{lllllllll}
\hline $\begin{array}{l}\text { Time } \\
(\mathbf{m i n})\end{array}$ & Abs & $\begin{array}{l}\text { Concentration } \\
(\boldsymbol{\mu g} / \mathbf{m l})\end{array}$ & D.F. & $\begin{array}{l}\text { Amount in } \\
\mathbf{5 m l}\end{array}$ & $\begin{array}{l}\text { Amount in } \\
\mathbf{9 0 0 m l}\end{array}$ & CLA & CDR & \%CDR \\
\hline 0 & 0 & 0 & 0 & 0 & 0 & 0 & 0 & 0 \\
2 & 0.0259 & 0.9087 & 10 & 0.0454 & 8.1783 & 0 & 8.1783 & 41.38 \\
4 & 0.0321 & 1.1263 & 10 & 0.0563 & 10.1821 & 0.0454 & 10.1821 & 51.52 \\
6 & 0.0498 & 1.7473 & 10 & 0.0873 & 15.7257 & 0.1017 & 15.8274 & 80.09 \\
8 & 0.0550 & 1.9298 & 10 & 0.0964 & 17.3682 & 0.189 & 17.5572 & 88.85 \\
10 & 0.0594 & 2.0842 & 10 & 0.1042 & 18.7578 & 0.2854 & 19.0432 & 96.37 \\
\hline
\end{tabular}

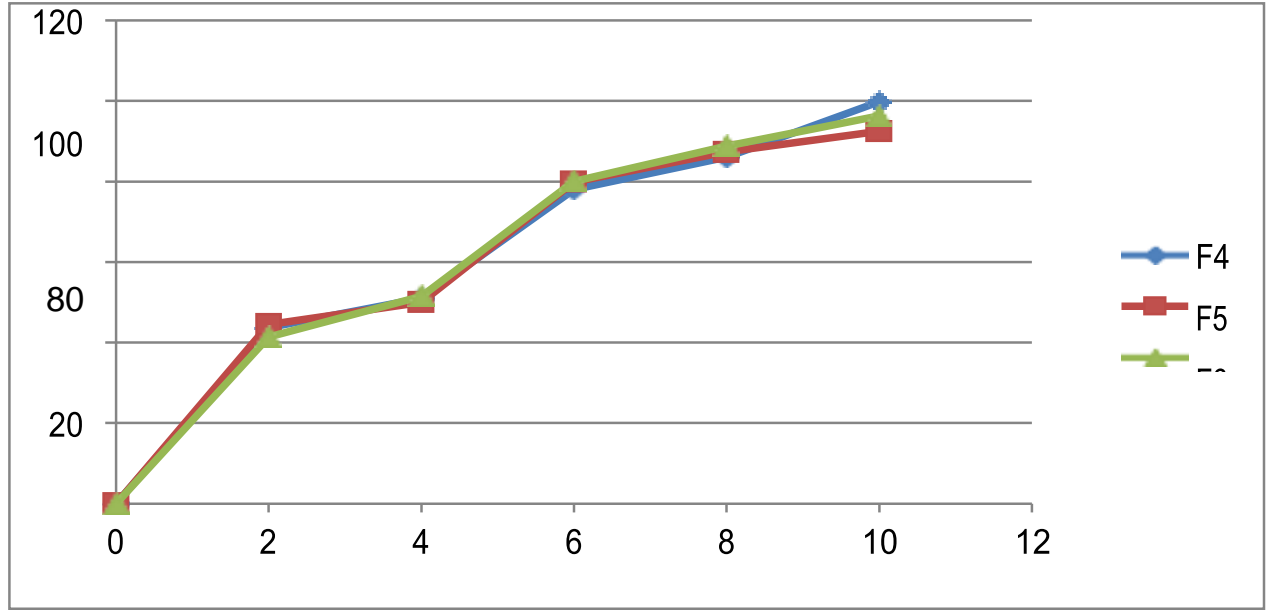

Figure 11: In vitro dissolution study of formulations F4, F5 and F6

Table 11: In vitro dissolution study of formulations F7

Weight of the tablet $=205 \mathrm{mg}$. Amount of drug present $=19.62 \mathrm{mg}$

\begin{tabular}{|c|c|c|c|c|c|c|c|}
\hline $\begin{array}{l}\text { Time } \\
\text { min }\end{array}$ & abs & $\begin{array}{l}\text { Concentration } \\
(\mu \mathrm{g} / \mathrm{ml})\end{array}$ & D.F & $\begin{array}{l}\text { Amount } \\
\text { in 900ml }\end{array}$ & CLA & CDR & \% CDR \\
\hline 0 & 0 & 0 & 0 & 0 & 0 & 0 & 0 \\
\hline 2 & 0.0281 & 0.9859 & 10 & 00492 & 8.8731 & 8.8731 & 45.22 \\
\hline 4 & 0.0312 & 1.0947 & 10 & 0.0547 & 9.8523 & 9.9015 & 50.46 \\
\hline 6 & 0.0514 & 1.8035 & 10 & 0.0901 & 16.2315 & 16.3354 & 83.25 \\
\hline 8 & 0.0539 & 1.8912 & 10 & 0.0945 & 17.0208 & 17.2148 & 87.74 \\
\hline 10 & 0.0571 & 2.0035 & 10 & 0.1001 & 18.0315 & 18.32 & 93.37 \\
\hline
\end{tabular}


Table 12: In vitro dissolution study of formulations F8

Weight of the tablet $=206 \mathrm{mg}$. Amount of drug present $=19.56 \mathrm{mg}$

\begin{tabular}{lllllllll}
\hline $\begin{array}{l}\text { Time } \\
(\mathbf{m i n})\end{array}$ & Abs & $\begin{array}{l}\text { Concentration } \\
(\boldsymbol{\mu g} / \mathbf{m l})\end{array}$ & $\begin{array}{l}\text { D.F. } \\
\text { Amount }\end{array}$ & $\begin{array}{l}\text { Amount } \\
\text { in 5ml }\end{array}$ & $\begin{array}{l}\text { CLA } \\
\text { in 900ml }\end{array}$ & CDR & \% CDR \\
\hline 0 & 0 & 0 & 0 & 0 & 0 & 0 & 0 & 0 \\
2 & 0.0301 & 1.0561 & 10 & 0.0528 & 9.5049 & 0 & 9.5049 & 48.59 \\
4 & 0.0334 & 1.1719 & 10 & 0.0585 & 10.5471 & 0.0528 & 10.6018 & 54.20 \\
6 & 0.0514 & 1.8035 & 10 & 0.0901 & 16.2315 & 0.1113 & 16.3428 & 83.55 \\
8 & 0.0550 & 1.9298 & 10 & 0.0964 & 17.3682 & 0.2014 & 17.5696 & 89.82 \\
10 & 0.0594 & 2.0842 & 10 & 0.1042 & 18.7578 & 0.2978 & 19.0556 & 97.42 \\
\hline
\end{tabular}

Table 13: In vitro dissolution study of formulations F9

Weight of the tablet $=205 \mathrm{mg}$. Amount of drug present $=19.46 \mathrm{mg}$

\begin{tabular}{lllllllll}
\hline $\begin{array}{l}\text { Time } \\
(\mathbf{m i n})\end{array}$ & Abs & $\begin{array}{l}\text { Concentration } \\
(\boldsymbol{\mu g} / \mathbf{m l})\end{array}$ & D.F. & $\begin{array}{l}\text { Amount } \\
\text { in 5ml }\end{array}$ & $\begin{array}{l}\text { Amount } \\
\text { in 900ml }\end{array}$ & CLA & CDR & \%CDR \\
\hline 0 & 0 & 0 & 0 & 0 & 0 & 0 & 0 & 0 \\
2 & 0.0301 & 1.0561 & 10 & 0.0528 & 9.5049 & 0 & 9.5049 & 48.84 \\
4 & 0.0371 & 1.3017 & 10 & 0.0650 & 11.7153 & 0.0528 & 11.7681 & 60.47 \\
6 & 0.0550 & 1.9298 & 10 & 0.0964 & 17.3682 & 0.1178 & 17.48 & 89.85 \\
8 & 0.0571 & 2.0035 & 10 & 0.1001 & 18.0315 & 0.2142 & 18.2457 & 93.76 \\
10 & 0.0614 & 2.1543 & 10 & 0.1077 & 19.3887 & 0.3143 & 19.703 & 101.24 \\
\hline
\end{tabular}

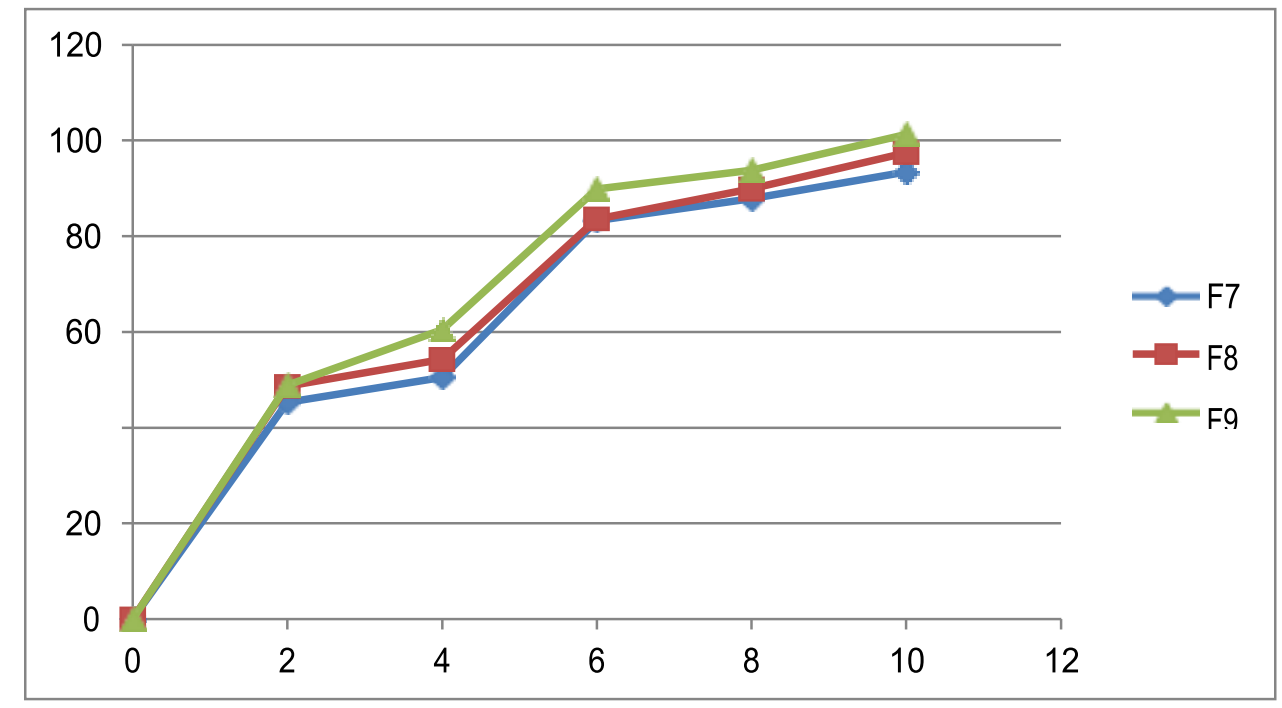

Figure 3: In vitro dissolution study of formulations F7, F8 and F9

\section{CONCLUSION}

Pantoprazole Sodium, the selective H2 blocker could be used to develop the FDT to release the drug in mouth and immediate absorption for the benefit of effective therapeutic action. The tablets prepared met the standard evaluation parameters with a slight deviation within the prescribed limits. The short term stability studies carried out were confirmative of the drug stability in the tablets during the present study. The disintegration studies revealed that the 
tablets prepared with crospovidone shown faster disintegration as compared to tablets prepared with croscarmilose sodium and sodium starch glycolate. Even the dissolution studies confirmed that the tablets prepared with crospovidone, shown faster drug release as compared to tablets prepared with croscarmilose sodium and sodium starch glycolate. From the above said it could safely concluded that the fast dissolving tablets of Pantoprazole Sodium prepared with crospovidone showed better disintegration time and the dissolution profile. Further it is advised that the same work should be confirmed for its therapeutic efficacy with the experimental and clinical trials.

\section{REFERENCES:}

1. Kumari S., Visht S. , Sharma PK. , Fast dissolving drug delivery system. Review article, J pharm Res. 2010:3(6):1449

2. Puttalingaiah L. , Kunchu K, Tamizh MT. , Fast disintegrating tablets : An overview of formulation, technology and evaluation . Res J, Pharm Bio chem. sci,2011:2(2):589-601.

3. Divate S, Kunchu K., Sockan G. N. Fast disintegrating tablets : An emerging trend Int. J. Pharm sci., Review and research. 2010:6(2):18-22.

4. Siddiqui MN., Garg G., Sharma PK. Fast dissolving tablets : preparation , characterization and evaluation ; An review . Int J. Pharm sci Review and Research 2010; 4(2):87-96.

5. Mallet L. caring for the elderly patient, J pharm assoc1996;36(11):628-35.

6. Bharawaj S. Jain S. Sharma S. Jat RC.Jain S. orally disintegrating tablets . A review drug invention today;2010:2(1):81-89.

7. Hirani JJ, Rathod DA, Vadalia KR. orally disintegrating tablets . A review tropical journal of pharmaceutical research 2009;8(2):161-72

8. Yourong S. Shicheng Y, Jeong SH. Kimura S. Park K. Orally fast disintegrating tablets; Development technologies, test masking and clinical studies. Critical reviews in therapeutic drug carrier systems 2004;21(6):433-75.

9. Deshpande KB. Orodispersible tablets :An overview of formulation and technology. Int J Pharm Biosci;2011:2(1)..

10. Lachman L. Lieberman HA. Kanig JL. The theory and practice of Ind pharmacy 3 ed Bombay: Varghese Publishing house 2006 67-83/

11. Pahwa R. Piplani M. Sharma PK. Kaushik D. Nanda S. Orally disintegrating tablets : Friendly to pediatrics and geriatrics. Scholars research library 2010;2(2):35-48.

12. Mohanchandran PS., Sindhumol PG., Kiran TS., Superdisintegrants: An overview Int. J. 
Pharm. Sci. Review and Research.2011;6(1):105-9

13. David EG. Armen HT. Ehrin JA and April WA. Principles of pharmacology 2nd edi Wolters Kluwer. India.2008:811-23.

14. Wise DL. Handbook of pharmaceutical controlled release Technology . Mercel Dekker India 2005: 435-40.

15. Iyer SR. Shivkumar R. Shiva P. Sajeeth cl. Formulation and evaluation of fast dissolving tablets of Resperidone solid dispersion, Int $\mathrm{J}$ Pharm Chem b Inclusion io sci 2013;3(2);388-397.

16. Dahima R. Bhatare D. Rathore D. Formulation and development of mouth dissolving tablets of a poorly water soluble drug using sublimation technique. Bulletin pharm Res 2012;2(1):5-9.

17. Biswanath S. Giri TK. Prperation and evaluation of rapidly disintegrating fast release tablets of Diazepam-hydroxypropyln- beta-cyclodextrin Inclusion complex pharmacology Pharm 2010;1:18-26.

18. Chowdary KPR. Aishwarya KNVR. Preparation and evaluation of fast dissolving tablets of paracetamol Employing superdisintegrants. Journal global trends Pharm sci 2013;4(4):1329-34.

19. Paul Y. Tyagi S. Singh B. Formulation and evaluation of oral dispersible Tablets of Zidovudine with different superdisintegrants. Int J. Corr Pharm Rev 2011;2(2):81-91.

20. Prajapati B. Kaur S. Roopini SA. Formulation and Evaluation of mouth dissolving sublingual tablets of Cimetidine to treat abdominal cramps. Int J. Pharm sci. Invention 2014;3(9):41-46.

21. Tomar N. Tomar M. Gulati N. Nagaich U. Formulation and Evaluation of Fast dissolving disintegrating Tablets of caffeine by using Effervescent. Formulation Approach scholars research library . Der Pharmacia Letter 2012; 4(5):1490-94.

22. Jain CP. Naruka PS. Formulation and Evaluation of Fast dissolving tablets of Valsartan. Int J. Pharm. sci. 2009;1(1):219-26.

23. Rangole U. Kawtikar PS. Sakarkar DM. Formulation and in-vitro evaluation of Rapidly Disintegrating tablets using Hydrochlorothiazide as a model drug Res J. Pharm. 2008;1(4):349-52.

24. Birajdar SM. Bhusnure OG. Mulaje SS, Formulation and evaluation of fast disintegrating Losartan Potassium Tablets by Formal Experimental Design, Int. J. Res. Pharm life sci 2014;3(5):1136-50. 
25. Rao NGR. Kumar M. Reddy MS. Kistayya C. Reddy BM. Development and Evaluation of Fast dissolving tablets of Fosinopril by sublimation method. Int. J. Pharm. sci. Res. 2012;4(4):230-35.

26. Peter R. Shashank NN. Shweta SKK. Shabaraya AR. Formulation and Evaluation of fast dissolving Tablets of Flunarizine Hydrochloride by sublimation method using sodium starch glycolate as superdisintegrant. Int. J. Pharm. Res. 2014;6(3):1085-95.

27. Chandir aRM. Venkateswarla , Bhowmik D. Kumar KPS. Chiranjiv, Jaykar B. Formulation ,Development and Evaluation of fast dissolving tablets of Carvedilol. T. Pharm. Res 2010;3:203-220. 